\title{
Integrate Cultures and Beliefs into Genealogy Software for Remote Communities in Borneo
}

\author{
Edwin Mit ${ }^{1}$, Cheah Wai Shiang ${ }^{2}$, Muhammad Asyraf Khairuddin ${ }^{3}$, and Noor Hazlini Borhan ${ }^{4}$ \\ Inst. of Social Informatics and Technological Innovations ${ }^{1,2}$ \\ Faculty of Computer Science and Information Technology ${ }^{3,4}$ \\ Universiti Malaysia Sarawak \\ Kota Samarahan, Malaysia \\ edwin@fit.unimas.my ${ }^{1}$,wscheah(@fit.unimas.my ${ }^{2}$, kmasyraf(@ $@$ fit.unimas.my ${ }^{3}$, bnhazlini(@ fit.unimas.my ${ }^{4}$
}

\begin{abstract}
The biggest challenge to precisely model the remote communities cultures in Borneo is to elicit the reliable documentary sources or historical data, as almost of it is not recorded. This is partly due to the accessibility to education, ICT technologies and the development of infrastructure was a bit late by the remote communities in Borneo. Therefore, the possible source of historical data is through oral tradition, which is have limited trace only to several generations back. In order to preserve their cultures and beliefs, a thorough study on remote communities is required in order to present a precise software architecture that can represent their cultures and beliefs in digital form. This paper will discuss the elicitation process and techniques on remote community cultures which related to marriage process, including preparations, events and beliefs before and during the marriage ceremony. These cultures are integrated into a proposed architecture of genealogy software for remote communities. The proposed genealogy software not only accommodates basic information about individuals, including births, marriages, and deaths but the most important component is the culture behind every event for historical records. The idea of this research is not only to preserve genealogy data, but the cultural of minority ethnic group of remote community in Borneo in a new genealogy software, so that the culture will not be extinct.
\end{abstract}

Keywords - culture; remote communities; software; genealogy; UML; formal VDM++

\section{INTRODUCTION}

Genealogy is the study of families and the tracing of their lineages and history. Genealogists use oral traditions, historical records, genetic analysis, and other records to obtain information about a family and to demonstrate kinship and pedigrees of its members. The results are often displayed in charts or written as narratives. Traditionally, the basic information needed to ensure correct identification of each person are place names, occupations, family names, first names, and dates. However, modern genealogists greatly expand this list, recognizing the need to place this information in its historical context in order to properly evaluate genealogical evidence and distinguish between same-name individuals [1]. However, the biggest challenge in remote communities of Borneo is to elicit the documentary sources or historical data as almost of it is not recorded. This is partly due to the accessibility to education, ICT technologies and the development of infrastructure was a bit late at the remote communities in Borneo. Therefore, the possible source of historical data is through oral tradition, which is have limited trace onlty to several generations back.

This project focuses on designing and developing genealogy software based on cultural model for remote community in Borneo [2]. There are number of cultures related to daily life of remote communities. However, at this stage of research, this project is working on marriage cultures of remote communities, which is very closely related to the proposed new family tree (genealogy) for remote community. This research will model the marriage processes such as prepreparations, and events during the marriage ceremony, then later define relationship of this marriage processes to the proposed genealogy architecture for remote community. The proposed new genealogy software will accommodate basic information about individuals, including births, marriages, and deaths and the most important is the story behind these activities/events for historical records. By integrating the cultures, in which it is an event that need to be recorded at particular point of time during the marriage ceremony, can help the communities to understand their cultures better. In other word, a particular event that occur during the marriage process (e.g., dance, poem, rhyme etc), or a natural events (e.g., heavy rain during marriage ceremony), indicate that the couple is blessed (e.g., rich, many children, etc). So far, this is difficult to proof, as most couple, may not remember natural events happened during their marriage, otherwise it is a major event. Therefore the proposed new genealogy for remote communities will provide the platform to record the events and consequences of the events. By doing this the young generation can learn/preserve why they need to perform a particular event, and what are the consequences if they do or they do not do it? Therefore, the unique idea behind this research project is not only to preserve genealogy data, but the new architecture of genealogy software that integrates cultures of minority ethnic group of remote community in Borneo so that the culture will not be extinct.

This research project use interview technique in order to collect documentary sources (e.g., legend or warrior, 